\title{
Article \\ Optimization of a Spin-Orbit Torque Switching Scheme Based on Micromagnetic Simulations and Reinforcement Learning
}

\author{
Roberto L. de Orio $^{1, * \mathbb{C}}$, Johannes Ender ${ }^{2}$, Simone Fiorentini ${ }^{2}{ }^{\circledR}$, Wolfgang Goes ${ }^{3}$, Siegfried Selberherr ${ }^{1}(\mathbb{D}$ and \\ Viktor Sverdlov ${ }^{2}$ \\ 1 Institute for Microelectronics, TU Wien, Gußhausstraße 27-29/E360, 1040 Vienna, Austria; \\ Selberherr@TUWien.ac.at \\ 2 Christian Doppler Laboratory for Nonvolatile Magnetoresistive Memory and Logic at the Institute for \\ Microelectronics, TU Wien, 1040 Vienna, Austria; ender@iue.tuwien.ac.at (J.E.); \\ fiorentini@iue.tuwien.ac.at (S.F.); sverdlov@iue.tuwien.ac.at (V.S.) \\ 3 Silvaco Europe Ltd., Cambridge PE27 5JL, UK; wolfgang.goes@silvaco.com \\ * Correspondence: orio@iue.tuwien.ac.at
}

check for updates

Citation: de Orio, R.L.; Ender, J.; Fiorentini, S.; Goes, W.; Selberherr, S.; Sverdlov, V. Optimization of a SpinOrbit Torque Switching Scheme Based on Micromagnetic Simulations and Reinforcement Learning. Micromachines 2021, 12, 443. https:// doi.org/10.3390/mi12040443

Academic Editor: Sumeet Kumar Gupta

Received: 12 March 2021 Accepted: 13 April 2021 Published: 15 April 2021

Publisher's Note: MDPI stays neutral with regard to jurisdictional claims in published maps and institutional affiliations.

Copyright: (c) 2021 by the authors. Licensee MDPI, Basel, Switzerland. This article is an open access article distributed under the terms and conditions of the Creative Commons Attribution (CC BY) license (https:/ / creativecommons.org/licenses/by/ $4.0 /)$.

\begin{abstract}
Spin-orbit torque memory is a suitable candidate for next generation nonvolatile magnetoresistive random access memory. It combines high-speed operation with excellent endurance, being particularly promising for application in caches. In this work, a two-current pulse magnetic field-free spin-orbit torque switching scheme is combined with reinforcement learning in order to determine current pulse parameters leading to the fastest magnetization switching for the scheme. Based on micromagnetic simulations, it is shown that the switching probability strongly depends on the configuration of the current pulses for cell operation with sub-nanosecond timing. We demonstrate that the implemented reinforcement learning setup is able to determine an optimal pulse configuration to achieve a switching time in the order of $150 \mathrm{ps}$, which is $50 \%$ shorter than the time obtained with non-optimized pulse parameters. Reinforcement learning is a promising tool to automate and further optimize the switching characteristics of the two-pulse scheme. An analysis of the impact of material parameter variations has shown that deterministic switching can be ensured for all cells within the variation space, provided that the current densities of the applied pulses are properly adjusted.
\end{abstract}

Keywords: spin-orbit torque MRAM; reinforcement learning; two-pulse switching scheme; magnetic field-free switching; machine learning

\section{Introduction}

Spin-transfer torque magnetoresistive random access memory (STT-MRAM) is currently the state-of-the-art MRAM technology, entering volume production at all major foundries [1-6]. It is an emerging nonvolatile technology suitable for future universal memory applications. One of its key advantages is that it is compatible with CMOS technology, so it can be straightforwardly embedded in circuits [7]. It is promising not only for standalone, but also for embedded memory applications as replacement of conventional volatile CMOS-based and nonvolatile flash memories in systems on chip. STT-MRAM can be integrated in a broad range of applications, from Internet-of-Things to automotive applications [3] and last level caches [8-10]. Recently, 1Gb standalone [11] and embedded STT-MRAM solutions $[2,4,12,13]$ have been reported and STT-MRAM operation with a timing of a few nanoseconds has been demonstrated [8]. However, in order to further reduce the timing below the nanosecond range, the required current density becomes quite large. This creates an important limitation, since large currents flowing through the thin tunnel oxide of a magnetic tunnel junction (MTJ) lead to reliability issues, reducing the MRAM endurance.

Spin-orbit torque (SOT) MRAM is a promising nonvolatile memory candidate outperforming STT-MRAM for ultra-fast operation [14]. In SOT-MRAM, the large current required 
for the writing operation does not flow through the MTJ. Switching is accomplished by applying a current through a heavy metal wire attached to the magnetic free layer (FL). Thus, it can operate with a sub-nanosecond timing retaining excellent endurance [15-17]. These properties make SOT-MRAM particularly interesting for nonvolatile replacement of the classical static random access memory (SRAM) used in caches. It should be pointed out, however, that deterministic SOT switching of a perpendicularly magnetized FL requires an external magnetic field [18]. Several field-free schemes have been proposed to circumvent this issue, usually at the cost of a more complex cell stack fabrication [15,16,19-23].

We consider an alternative field-free scheme, in which a purely electrical control of the switching process is realized by applying current pulses to two orthogonal heavy metal wires [24]. A proper configuration of the current pulses is able to reverse the perpendicularly magnetized FL [25]. Nevertheless, an interesting question arises: how can one determine a pulse sequence that leads to optimal switching? Searching for such a pulse sequence requires a very large number of experiments and/or simulations. Ideally, this task can be outsourced to an algorithm and performed in a guided and automated way.

Machine learning (ML) has been increasingly applied to the solution of physics-based problems [26] and has already been used to solve fundamental micromagnetic problems, such as the computation of the magnetization dynamics of a thin film [27] and of the magnetic microstructure of a single magnetic body [28]. Recently, an ML model was applied to identify the regime of field-free SOT switching as a function of the magnitude of the applied current density, the nanomagnet size, and the interfacial Dyzaloshinskii-Moriya interaction [29]. The two most used ML approaches are supervised and unsupervised learning. In turn, another sub-branch of ML so-called reinforcement learning (RL) has gained interest $[30,31]$. Big RL breakthroughs were achieved lately using games like chess or Go [32], but this type of learning algorithm has also been successfully applied for physics-based problems.

The RL principle of operation is based on an agent and an environment, in such a way that the agent interacts with the environment and learns how to act or take decisions to achieve a specific state or goal [30]. In other words, the agent interacts with the environment by performing actions that cause the environment to move from one state to another. Once the environment moves to a new state, it informs about its state and returns a reward to the agent. Based on this information, the agent can decide to take actions to maximize the cumulative reward received over time. During this process, the agent learns how to achieve the given objective.

In this work, we combine the two-current pulse switching scheme with an RL algorithm to optimize the switching of a spin-orbit torque memory cell. We demonstrate that the reinforcement learning implementation can find an optimal sequence and timing for the current pulses in order to achieve faster switching in comparison to a conventional combination of pulse parameters.

\section{Spin-Orbit Torque Memory Cell and Switching Dynamics}

The two-pulse switching scheme for a SOT memory cell is depicted in Figure 1. The cell is formed by growing a perpendicularly magnetized FL on top of a heavy metal wire (NM1), where a first current pulse is applied to generate the initial SOT on the FL. On the right part of the cell, a second, orthogonal heavy metal wire (NM2) is placed on top of the $\mathrm{FL}$, and a second current pulse is applied through it. The SOT generated due to this second pulse acts on the FL to complete the magnetization switching of the memory cell [33]. The $\mathrm{NM} 1 / \mathrm{FL} / \mathrm{NM} 2$ stack composes the structural part used for the writing operation of the memory cell. In the left part of the cell, next to the SOT writing stack, an MTJ is grown on top of the FL, which is required for carrying out the reading operation of the memory cell via measurement of the tunneling magnetoresistance. 


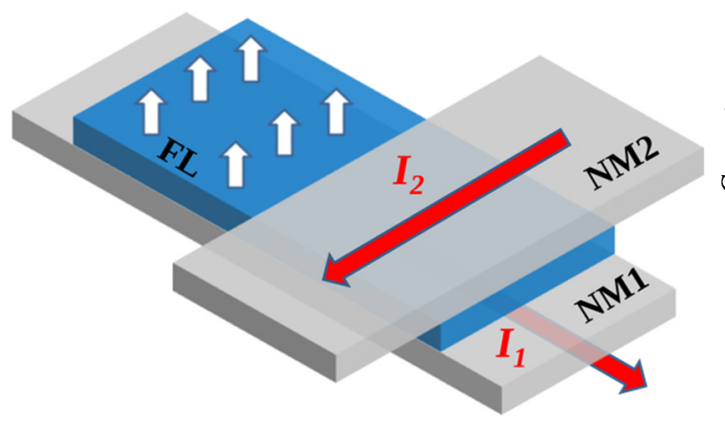

(a)

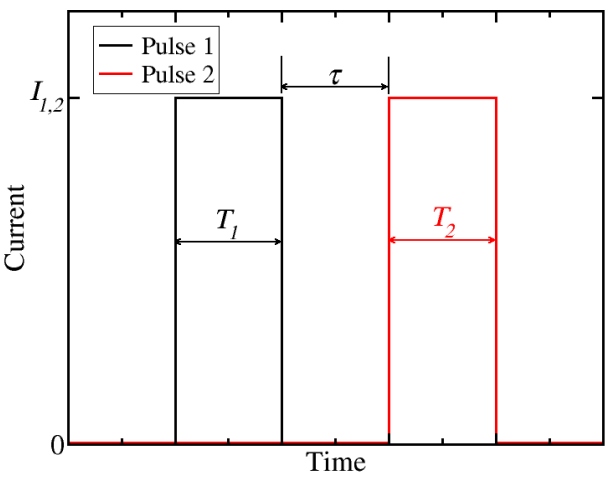

(b)

Figure 1. (a) SOT-MRAM cell for switching based on (b) two orthogonal current pulses. Pulse 1 is applied to the NM1 wire and Pulse 2 is applied to the NM2 wire. $I_{1,2}$ is the current amplitude and $T_{1,2}$ is the width of the first/second pulse. $\tau$ represents the delay or overlap between the pulses.

In order to carry out micromagnetic simulations of the two-pulse SOT switching scheme, the magnetization dynamics is described by the Landau-Lifshitz-Gilbert (LLG) equation

$$
\begin{aligned}
& \frac{\partial \mathbf{m}}{\partial t}=-\gamma \mu_{0} \mathbf{m} \times \mathbf{H}_{\mathbf{e f f}}+\alpha \mathbf{m} \times \frac{\partial \mathbf{m}}{\partial t} \\
& -\gamma \frac{\hbar}{2 e} \frac{\theta_{S H} j_{1}}{M_{S} d}[\mathbf{m} \times(\mathbf{m} \times \mathbf{y})] \Theta\left(t, T_{1}\right) \\
& +\gamma \frac{\hbar}{2 e} \frac{\theta_{S H} j_{2}}{M_{S} d}[\mathbf{m} \times(\mathbf{m} \times \mathbf{x})] \Theta\left(t, T_{1}, T_{2}\right)
\end{aligned}
$$

where $\mathbf{m}$ is the normalized magnetization, $\gamma$ is the gyromagnetic ratio, $\mu_{0}$ is the vacuum permeability, $\alpha$ is the Gilbert damping factor, and $M_{S}$ is the saturation magnetization. $\mathbf{H}_{\text {eff }}$ is an effective magnetic field, which includes the exchange field, the uniaxial perpendicular anisotropy field, the demagnetization field, the current-induced field, and the stochastic thermal field at $300 \mathrm{~K}$. The last two terms on the right-hand side of the LLG equation describe the SOT generated by the applied current pulses through the NM1 and the NM2 wire, respectively, where $e$ is the elementary charge, $\hbar$ is the reduced Plank constant, $\theta_{S H}$ is an effective Hall angle, $j_{1,2}$ is the current density of the first/second pulse, $d$ is the FL thickness, and $\Theta(\cdot)$ is a function which determines when each pulse is active.

Equation (1) is solved numerically using a micromagnetic simulation software developed in-house [34] based on the finite difference method. The simulation parameters are given in Table 1.

Table 1. Simulation parameters. Heavy metal wires of b-tungsten and a magnetic FL of CoFeB on $\mathrm{MgO}$ are assumed [18].

\begin{tabular}{cc}
\hline Parameter & Value \\
\hline Saturation magnetization, $M_{S}$ & $1.1 \times 10^{6} \mathrm{~A} / \mathrm{m}$ \\
Exchange constant, $A$ & $1.0 \times 10^{-11} \mathrm{~J} / \mathrm{m}$ \\
Perpendicular anisotropy, $K$ & $8.4 \times 10^{5} \mathrm{~J} / \mathrm{m}^{3}$ \\
Gilbert damping factor, $\alpha$ & 0.035 \\
Spin Hall angle, $\theta_{S H}$ & 0.3 \\
Thermal stability factor, $\Delta$ & 45 \\
Free layer dimensions & $40 \mathrm{~nm} \times 20 \mathrm{~nm} \times 1.2 \mathrm{~nm}$ \\
NM1: $w_{1} \times l$ & $20 \mathrm{~nm} \times 3 \mathrm{~nm}$ \\
NM2: $w_{2} \times l$ & $20 \mathrm{~nm} \times 3 \mathrm{~nm}$ \\
\hline
\end{tabular}

\section{Reinforcement Learning for the Two-Pulse Spin-Orbit Torque Switching}

Figure 2 shows the RL setup implemented for performing the learning experiments with the two-pulse switching scheme. The environment consists of our in-house tool, 
which provides the simulation of the memory cell switching and returns the current state of the simulation together with a reward after every iteration. The used deep Qnetwork (DQN) algorithm [31] incorporates a neural network to approximate a function for mapping states to actions. An existing Python library providing the RL capabilities has been employed [35]. Here, the goal of our RL implementation is to determine the pulse configuration which results in the shortest switching time, defined as the time when the perpendicular component of the magnetization vector reaches -0.5 , i.e., $m_{z}=-0.5$.

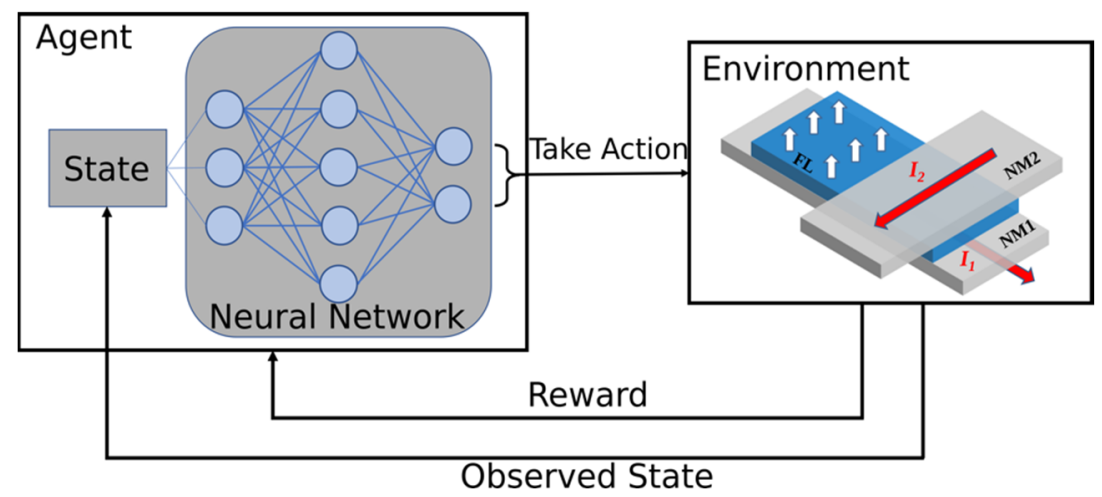

Figure 2. Reinforcement learning setup for the two-pulse switching scheme. The micromagnetic simulation of the memory cell provides the environment, with which the agent interacts and takes actions to achieve the fastest magnetization switching.

The state vector returned from the environment after every iteration consists of 11 variables: the average of the three magnetization vector components $\left(m_{x}, m_{y}, m_{z}\right)$, the difference of each component to the previous iteration $\left(\Delta m_{x}, \Delta m_{y}, \Delta m_{z}\right)$, the average component of the effective magnetic field $\left(H_{\text {eff }, x}, H_{\text {eff }, y}, H_{\text {eff } z z}\right)$, and two variables indicating whether the first and the second pulse are active or not. Based on the state information, the learning agent deduces which action to take. It is important that the dynamics of the magnetization vector, given by $\left(\Delta m_{x}, \Delta m_{y}, \Delta m_{z}\right)$, is taken into account, so the direction in which the magnetization is moving is known. In this way, the agent can decide on the best action to take to drive the switching as fast as possible. Our setup allows the agent to take four different actions, namely, setting both pulses off, setting both pulses on, turn the first pulse on with the second off, or turn the first pulse off and the second pulse on. If a pulse is on, it means that current has been applied to the corresponding heavy metal wire and a spin torque is applied to the magnetization of the FL.

The rewarding scheme is critical for the RL approach, because it is the main factor which leads the learning algorithm in the right direction and the agent to select the best actions to achieve the target. The reward is an integer value returned by the environment, indicating whether the actions performed by the agent were good or bad. For the SOT switching, the rewarding scheme is chosen such that a shorter switching time corresponds to a higher reward, since the RL algorithm tries to maximize the cumulative reward during the learning process. Here, a reward of -1 is given for every simulation step in which the target, $m_{z}=-0.5$, has not been reached yet. We define $t_{\max }=1 \mathrm{~ns}$ as an upper limit for the simulation time. If the target is not reached within this time, the learning episode is terminated and a new one is started. On the other hand, if the target is reached at a time $t_{\text {final }}$ before $t_{\text {max }}$, a positive reward of $\left(t_{\max }-t_{\text {final }}\right) / \Delta t$ is returned, where $\Delta t$ is the simulation time-step. In this way, the rewarding scheme is a complementary measure of the number of time-steps required to reach the switching. The smaller the number of time-steps needed to switch, the shorter the switching time is and, therefore, the larger the reward is. 


\section{Results and Discussion}

\subsection{Numerical Simulations}

Micromagnetic simulations of the switching dynamics of the two-pulse SOT scheme, as described in Section 2, were carried out. We start by investigating the impact of the pulse configuration on the magnetization dynamics. In particular, the current densities of the first and the second current pulse are fixed at $j_{1}=2.7 \times 10^{12} \mathrm{~A} / \mathrm{m}^{2}$ and $j_{2}=1.3 \times 10^{12} \mathrm{~A} / \mathrm{m}^{2}$, respectively, while the pulse durations $T_{1}$ and $T_{2}$ can be modified (c.f. Figure 1). A perfect synchronization between the pulses is considered, i.e., the second current pulse is turned on immediately after the first pulse is turned off. Thus, there is no delay or overlap between the pulses $(\tau=0)$. This constraint will be lifted in Section 4.2, where the results of the RL approach are discussed.

Figure 3 shows the perpendicular component of the magnetization $\left(m_{z}\right)$ as a function of time for different widths of the first current pulse, while the second pulse width is kept fixed at $T_{2}=100 \mathrm{ps}$. In order to account for the thermal spread resulting from the stochastic thermal field at room temperature, a total of 50 realizations are considered for each simulation condition. The curves shown in Figure 3 represent the average of these 50 realizations. One can clearly see that, depending on the width of the first pulse, the magnetization dynamics changes significantly, and so does the switching behavior. Here, the pulse sequence and the timing lead to successful magnetization reversal, when the width of the first pulse is short, while switching does not occur for larger values of $T_{1}$.

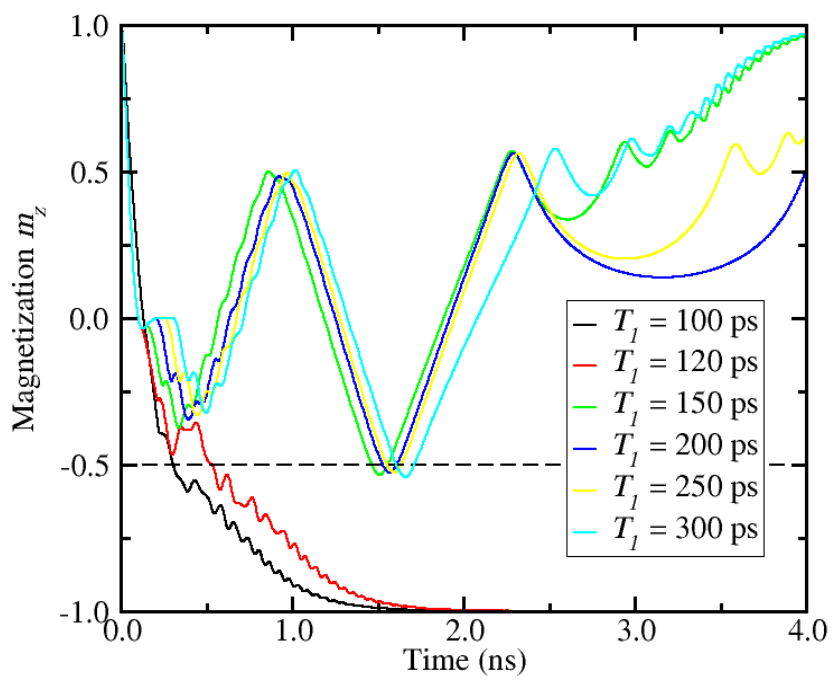

Figure 3. Perpendicular component of the magnetization vector (average of 50 realizations) as a function of time for various durations of the first pulse, $T_{1}$. The simulation parameters are found in Table 1 and $j_{1}=2.7 \times 10^{12} \mathrm{~A} / \mathrm{m}^{2}, j_{2}=1.3 \times 10^{12} \mathrm{~A} / \mathrm{m}^{2}$, and $T_{2}=100 \mathrm{ps} . j_{1}$ and $T_{1}$ are the current density and the duration of the first pulse, respectively, and $j_{2}$ and $T_{2}$ are the current density and the duration of the second pulse (c.f. Figure 1b). The dashed line represents the switching threshold.

Next, we reverse the analysis and fix the first current pulse width at $T_{1}=150 \mathrm{ps}$, while the width of the second current pulse is varied. The resulting magnetization dynamics is shown in Figure 4. As in the previous results, switching is obtained depending on the value of $T_{2}$. In contrast to the previous scenario, successful switching is observed as the second pulse width becomes longer.

The above results suggest that the configuration of the pulse sequence has an important impact on the switching characteristics of the cell, in such a way that variations of the pulse configuration can lead to either switching or non-switching schemes. To further understand this impact, we performed simulations for various combinations of pulses and evaluate the switching probability. The results are shown in Figure 5, which plots the switching probability as a function of the first and the second pulse width. In general, for short values of $T_{2}$ ( $\leq 150 \mathrm{ps}$ ), the switching probability depends largely on the first pulse 
width, i.e., it depends on the particular pulse sequence and small changes of the pulses can yield successful or non-successful magnetization switching. In turn, increasing $T_{2}$ beyond $\sim 200 \mathrm{ps}$, the switching probability tends to 1 , becoming practically insensitive to the duration of the first pulse.

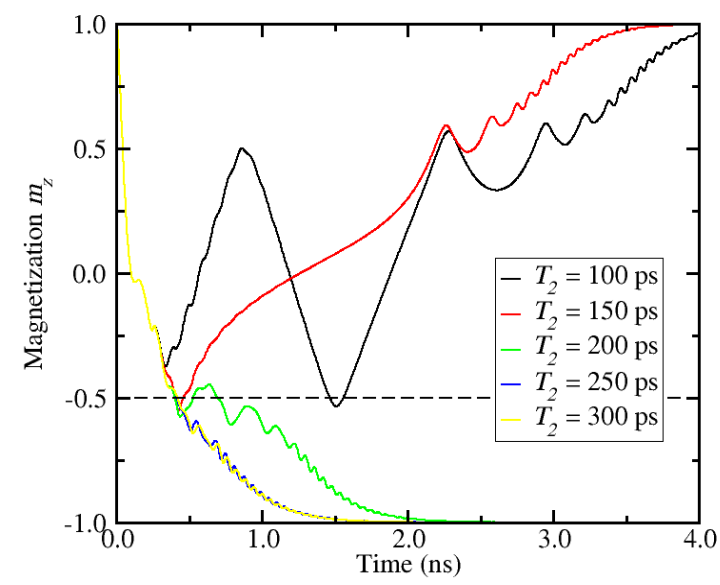

Figure 4. Switching dynamics for different values of the second pulse duration $T_{2}$ for a fixed first pulse width $T_{1}=150 \mathrm{ps}$. The dashed line represents the switching threshold.

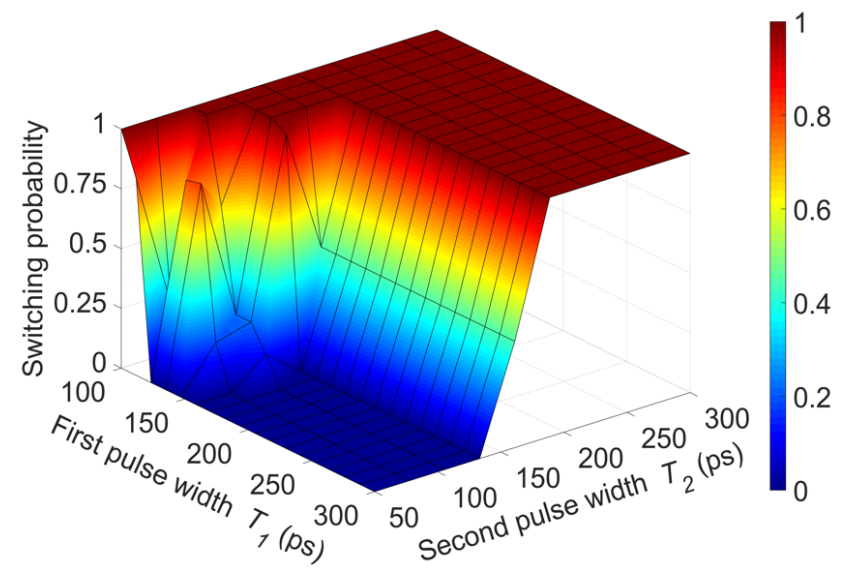

Figure 5. Switching probability as a function of the first and the second current pulse widths, $T_{1}, T_{2}$. For short pulse widths, precise pulse schemes are required to obtain deterministic switching.

From the previous analysis, we are able to determine pulse parameters that lead to deterministic switching of the memory cell. However, this does not guarantee that these parameters produce fast switching. Now we would like to find the pulse sequence which leads to the fastest possible switching. In order to accomplish that, we have to evaluate many more combinations of pulse sequences than those considered before. It should be pointed out that the previous results were obtained by manually running a total of 180 micromagnetic simulations. Considering that 50 realizations (due to the stochastic thermal field) are carried out for each pulse sequence combination, the number of switching simulations increases to 9000 , even though delays or overlaps between the pulses are still not considered. Thus, taking into account all possible variations of pulse parameters results in an exponential increase of the required number of simulations, which makes a manual optimization of the switching intractable. Here, the RL setup described in Section 3 is extremely useful, offering a powerful methodology for searching the fastest switching condition in a guided way. 


\subsection{Reinforcement Learning Experiments}

$\mathrm{RL}$ is applied with the goal of achieving the fastest magnetization switching, namely to achieve the shortest switching time, which is determined by the time when the condition $m_{z}=-0.5$ is reached. The agent searches for a pulse sequence and combination of the first and the second pulse duration, $T_{1}, T_{2}$, which lead to the shortest switching time. The actions performed by the agent (c.f. Figure 2) have been restricted to facilitate the learning process, thus it can switch on and off each pulse individually. However, the pulse synchronization constraint of the previous section is now relaxed, so that the current pulses are allowed to overlap or be delayed. The minimum pulse width is limited to $100 \mathrm{ps}$ and the amplitude of the pulse is fixed to $130 \mu \mathrm{A}$ and $100 \mu \mathrm{A}$ for the first and the second current pulse, respectively. A learning episode is finished once $m_{z}=-0.5$ or the time has reached 1 ns.

The results of the learning process of our RL setting are shown in Figures 6 and 7, respectively. Figure 6 reports the switching time over the course of the learning period for 20 independent learning runs, where each run encompasses $10^{6}$ learning steps. During an initial exploration phase, the action selection by the agent is not greedy, i.e., an action is not selected with the purpose of accumulating the highest reward, but the agent takes a random action to explore the state-action space. Furthermore, different random seeds are used for initializing the neural network weights. A general trend can, however, be observed, which is the reduction of the switching time as the number of learning steps increases. Initially, the switching times are distributed around $400-500 \mathrm{ps}$, but as the number of learning steps increases, several runs reach switching times in the 200-300 ps range.

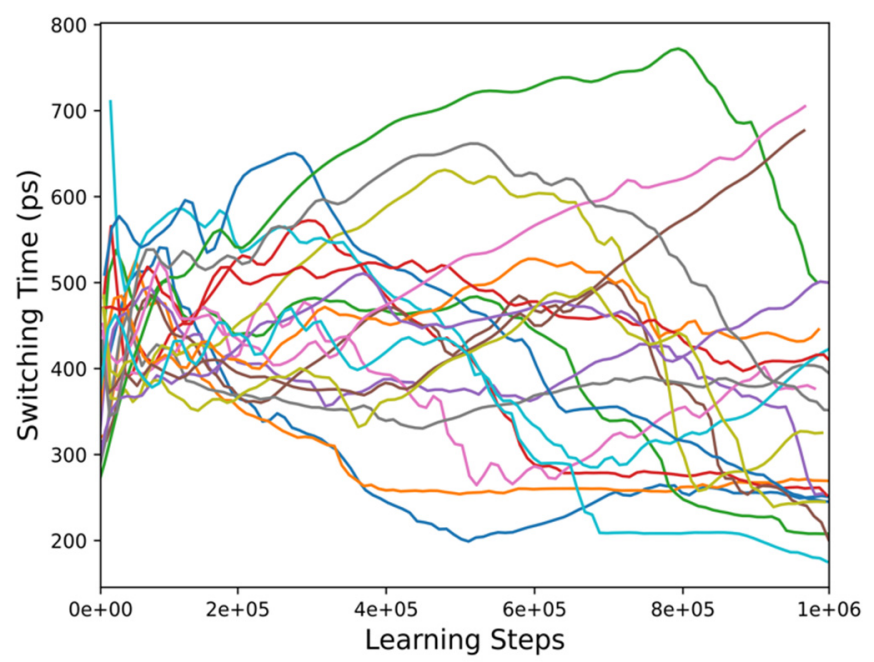

Figure 6. Switching time over the course of the learning period of 20 independent runs. As the number of learning steps increases, there is a trend towards switching time reduction.

The switching time decrease with the learning progress can be better visualized in Figure 7, which shows the mean switching time and the reward as a function of the number of learning steps of the six best learning runs. First, an increase of the switching time is observed, which is a consequence of the initial focus on exploration of the state-action space previously mentioned. Then, over the course of $10^{6}$ learning steps, the mean switching time reduces to around $240 \mathrm{ps}$. The direct relationship between the switching time and the accumulated reward is readily demonstrated in Figure 7. As the switching time decreases, the accumulated reward increases, indicating that the agent has learned a better policy to select actions which can switch the memory cell faster. It should be pointed out that single runs were able to achieve an even better policy, which resulted in a minimum switching time of about $146 \mathrm{ps}$. 


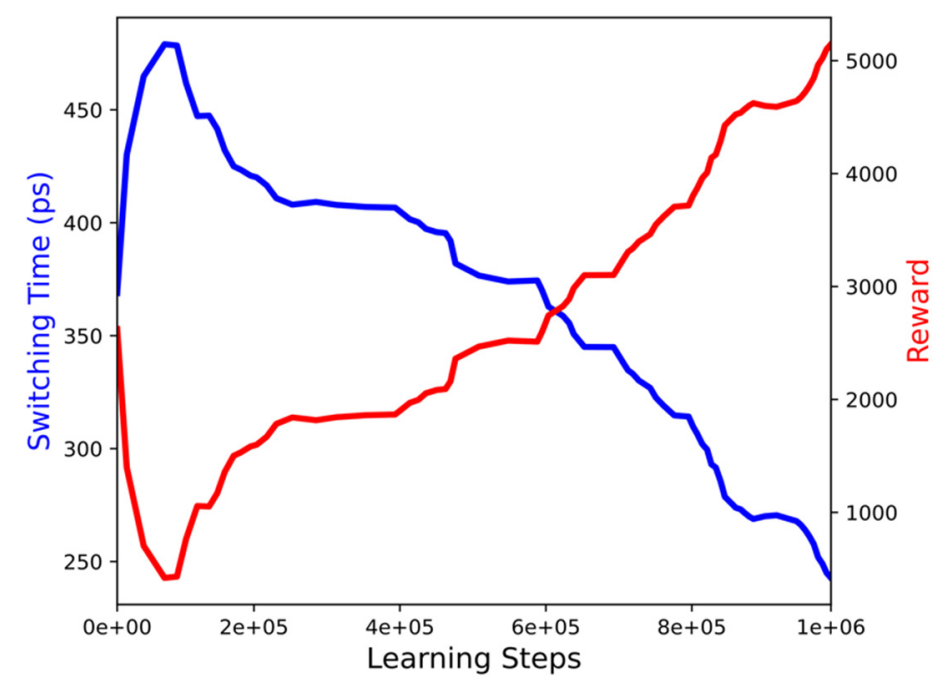

Figure 7. Learning curve showing the mean switching time and reward over $10^{6}$ time steps. It shows that, the faster the cell switches, the larger is the accumulated reward during learning.

The pulse configuration learned by the DQN algorithm and the resulting magnetization dynamics are shown in Figure 8. The current pulses through the NM1 and the NM2 wire are turned on simultaneously right in the beginning of the simulation. After $100 \mathrm{ps}$, the first pulse is turned off and the magnetization component $m_{z}$ drops below the -0.5 threshold. Once this threshold is achieved, no further action is taken and the second current pulse is kept active for the rest of the simulation. This generates a SOT which acts on the FL under the NM2 wire, resulting in an average perpendicular magnetization component of about -0.8 . Thus, the magnetization of the FL is not fully reversed to -1 . This demonstrates the importance of the rewarding scheme and the general setup of the RL experiment. As the RL agent was rewarded for finishing the learning episode as fast as possible and the episode was considered finished as soon as the -0.5 threshold was reached, the agent learned how to achieve the threshold and did not take any action afterwards.
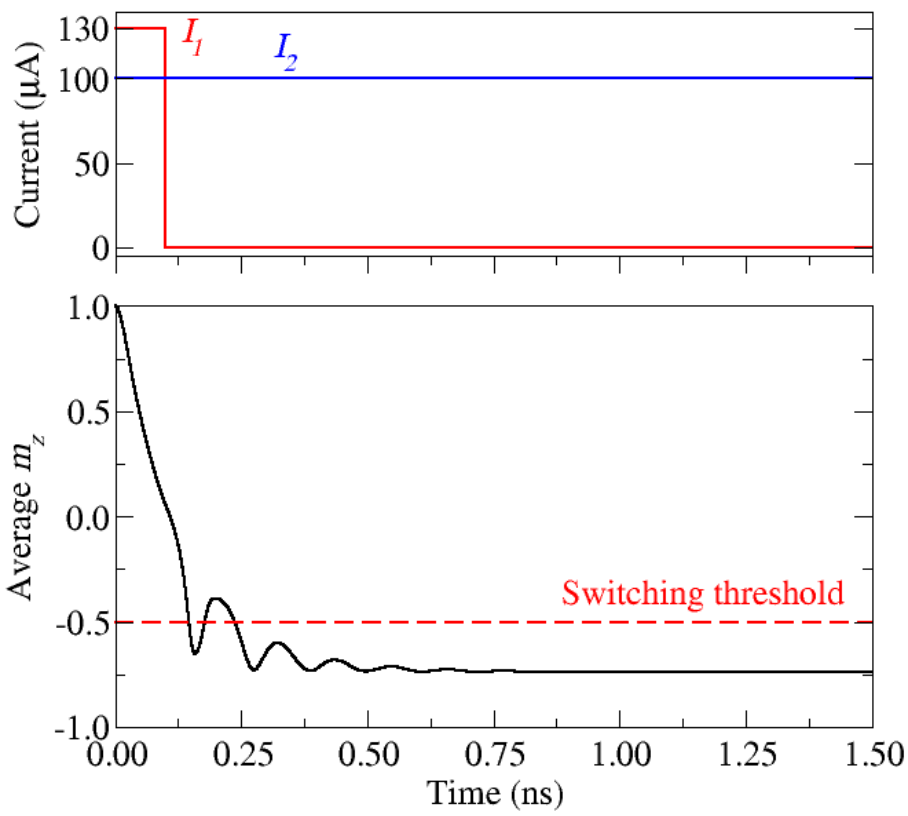

Figure 8. Pulse sequence learned by the DQN agent. $I_{1}$ is the current amplitude of the first pulse applied to the NM1 wire and $I_{2}$ is the current amplitude of the second pulse applied to the NM2 wire. 
Figure 9 shows the dynamics of the magnetization component $m_{z}$ considering different variations of the learned pulse configuration. In the learned model, the second pulse is now switched off after $m_{z}=-0.5$ is reached, which guarantees that the magnetization reversal is completed. The variations consisted of extending the first pulse and/or delaying the second pulse. A comparison of the magnetization dynamics with the learned model is given in Figure 9. One can observe that the learned configuration (black curve) leads indeed to the fastest switching. In turn, in the scenario with a longer first pulse, for which the pulses are almost perfectly overlapping, switching does not occur (red curve). The modified pulse sequences, represented by the green and blue curves, also lead to switching of the cell, however with longer switching times.

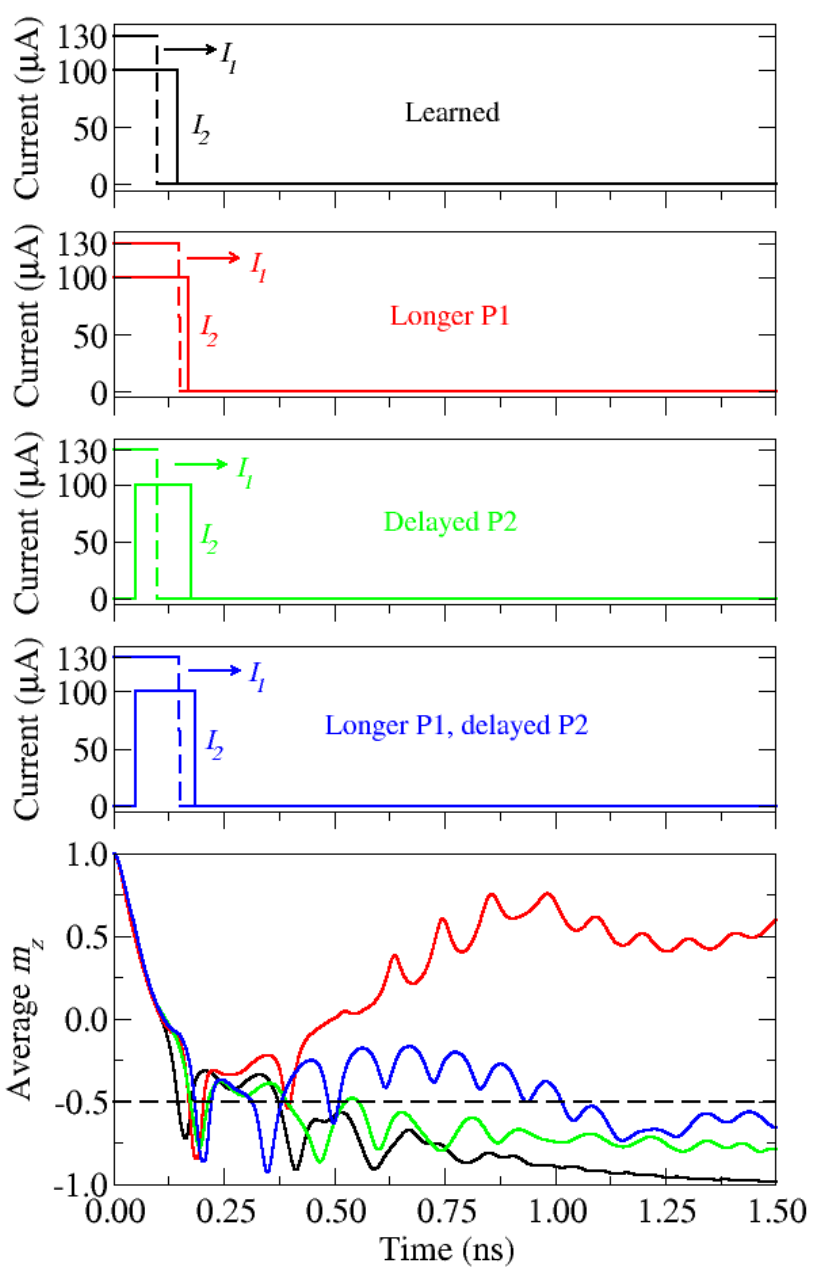

Figure 9. Comparison of different pulse configurations. The learned model is compared with modified ones. The learned pulse configuration leads to the fastest switching. $I_{1}$ is the current amplitude of the first pulse, P1, applied to the NM1 wire and $I_{2}$ is the current amplitude of the second pulse, P2, applied to the NM2 wire. The dashed line represents the switching threshold.

The robustness of the switching for the learned scheme is confirmed in Figure 10, for which 50 realizations under influence of the stochastic thermal field are reported. The variations between the different realizations are small and all of them switch, which shows that the learned scheme results in reliable and deterministic switching. It should be pointed out that, while the RL approach was able to find a scheme for which the switching time is $146 \mathrm{ps}$, the minimum switching time obtained from the previous manual configuration of the pulse was around $300 \mathrm{ps}$. This demonstrates the potential of the RL tool in combination with micromagnetic simulation for optimizing the two-pulse SOT switching scheme. 


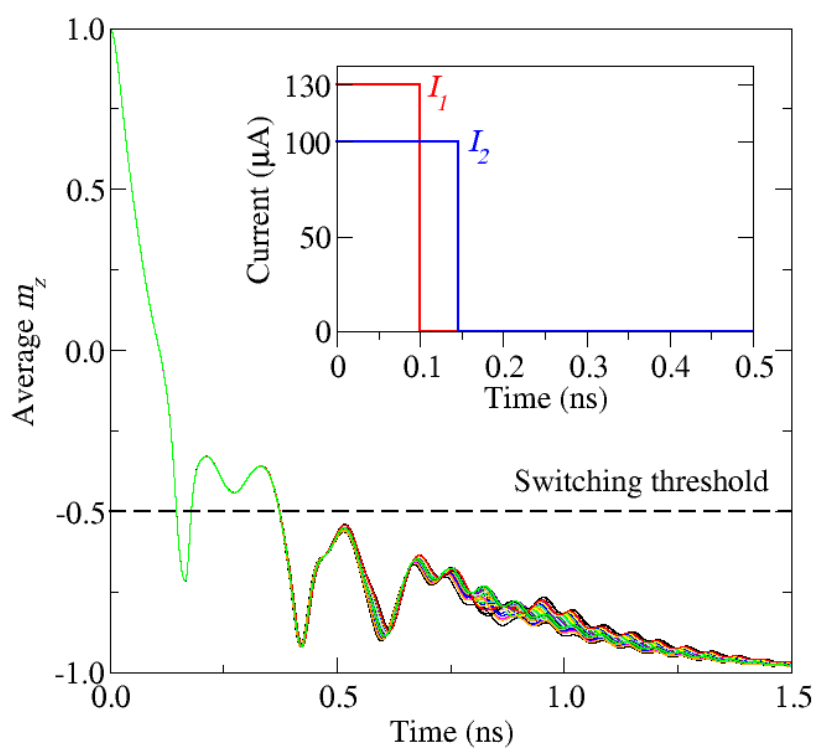

Figure 10. z-component of the magnetization of 50 switching realizations using the switching scheme found by the RL algorithm shown in the inset. $I_{1}$ is the current amplitude of the first pulse and $I_{2}$ is the current amplitude of the second pulse.

\subsection{Impact of Parameter Variations}

Although the fastest switching condition has been determined, variations of the pulse timing and/or of the process and material parameters of the magnetic FL can lead to slower or even non-deterministic switching. Thus, we now consider the impact of variations of the saturation magnetization and the anisotropy energy on the switching scheme.

Figure 11 shows the $\mathrm{x}, \mathrm{y}$, and $\mathrm{z}$ components of the magnetization vector as a function of time for $K=8.8 \times 10^{5} \mathrm{~J} / \mathrm{m}^{3}$ and $M_{S}=1.05 \times 10^{6} \mathrm{~A} / \mathrm{m}$, which represent a variation of $5 \%$ in relation to the nominal parameter values. In this case the cell does not switch and, more importantly, one can observe that the perpendicular component of the magnetization $\left(m_{z}\right)$ does not reduce below 0.7. This means that the SOT generated by the applied current density of the first pulse $\left(j_{1}=2.7 \times 10^{12} \mathrm{~A} / \mathrm{m}^{2}\right)$ is too weak to trigger the magnetization reversal. This can be explained by the fact that the variation of material parameters can change the critical current density for SOT switching. The above parameters lead to an increase of the critical current density, so that it becomes larger than the applied one. Thus, in order to switch this particular cell, the applied current has to be increased.

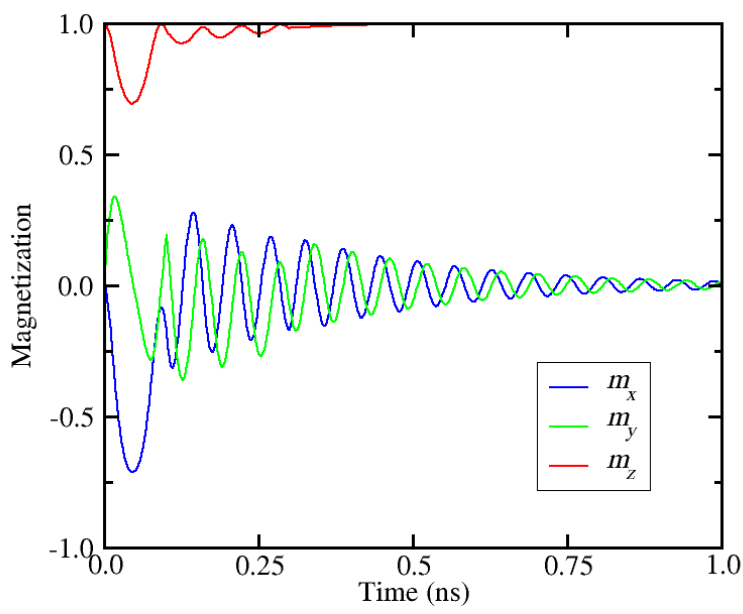

Figure 11. Magnetization dynamics for a cell with 5\% variation of the perpendicular anisotropy energy and saturation magnetization in relation to the nominal values. The current density of the first pulse $\left(2.7 \times 10^{12} \mathrm{~A} / \mathrm{m}^{2}\right)$ is smaller than the critical current density, so the cell does not switch. 
Considering that different material parameter variations happen concurrently, one should expect that different cells of the same design undergoing the same fabrication process can require different current densities to trigger switching. Figure 12 shows the required current density for the first pulse to guarantee deterministic switching for various combinations of saturation magnetization and anisotropy energy. For $10 \%$ variation of the parameters, the minimum switching current density varies from $1.0 \times 10^{12} \mathrm{~A} / \mathrm{m}^{2}$ to about $3.0 \times 10^{12} \mathrm{~A} / \mathrm{m}^{2}$. These results indicate that, in order to switch all cells within the parameter spread, a current density of at least $3.0 \times 10^{12} \mathrm{~A} / \mathrm{m}^{2}$ has to be applied for the first pulse.

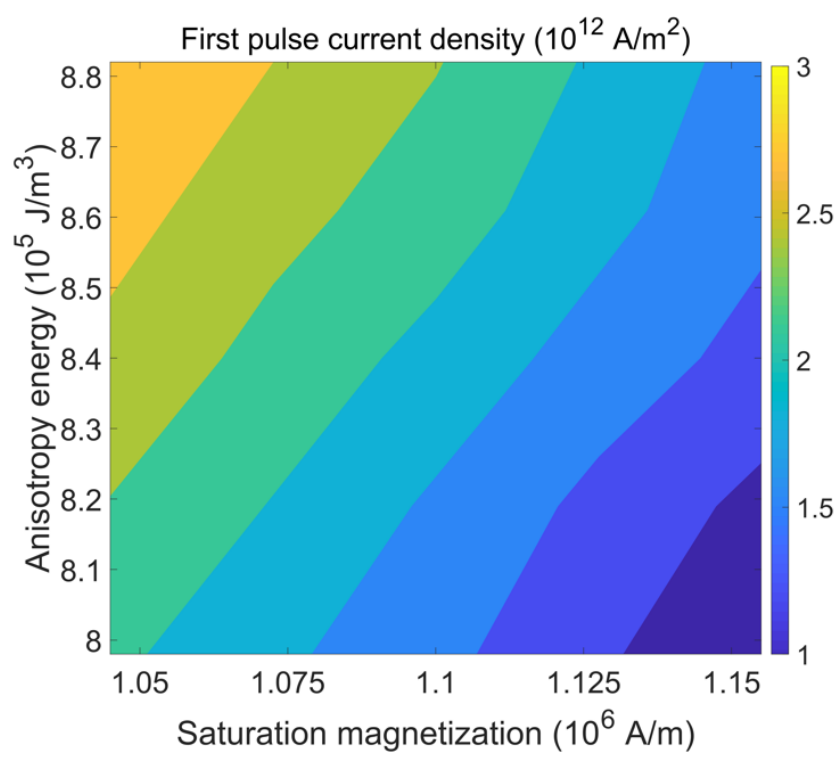

Figure 12. Minimum current density required for the first pulse to trigger reliable magnetization switching for cells with different combinations of saturation magnetization and anisotropy energy. The lowest current density is $1.0 \times 10^{12} \mathrm{~A} / \mathrm{m}^{2}$ (lower right corner) and the highest value is about $3.0 \times 10^{12} \mathrm{~A} / \mathrm{m}^{2}$ (upper left corner). The current density of the second pulse is $1.3 \times 10^{12} \mathrm{~A} / \mathrm{m}^{2}$ and both pulse durations are set to 200 ps.

Next, the required current density for the second pulse is determined, as shown in Figure 13. We consider four combinations of anisotropy energy and saturation magnetization, denominated $C 1$ to $C 4$, which cover the variation space of Figure $12: K=8.8 \times 10^{5} \mathrm{~J} / \mathrm{m}^{3}$, $M_{S}=1.05 \times 10^{6} \mathrm{~A} / \mathrm{m}(\mathrm{C} 1), K=8.8 \times 10^{5} \mathrm{~J} / \mathrm{m}^{3}, M_{S}=1.16 \times 10^{6} \mathrm{~A} / \mathrm{m}(\mathrm{C} 2), K=8.0 \times 10^{5} \mathrm{~J} / \mathrm{m}^{3}$, $M_{S}=1.05 \times 10^{6} \mathrm{~A} / \mathrm{m}(\mathrm{C} 3)$, and $K=8.0 \times 10^{5} \mathrm{~J} / \mathrm{m}^{3}, M_{S}=1.16 \times 10^{6} \mathrm{~A} / \mathrm{m}(\mathrm{C} 4)$, where $\mathrm{C} 1$ and $\mathrm{C} 4$ correspond to the two extreme cases, upper left and lower right corner, respectively, of Figure 12. In order to ensure 100\% switching, the minimum current density required for the second pulse is about $1.0 \times 10^{12} \mathrm{~A} / \mathrm{m}^{2}$.

The above analysis has allowed us to determine the minimum settings which guarantee $100 \%$ switching in the presence of cell-to-cell variations. Applying $j_{1}=3.0 \times 10^{12} \mathrm{~A} / \mathrm{m}^{2}$ and $j_{1}=1.3 \times 10^{12} \mathrm{~A} / \mathrm{m}^{2}$, the average switching realizations from parallel to anti-parallel (P$\mathrm{AP}$ ) as well as from anti-parallel to parallel (AP-P) configuration are reported in Figure 14, for the parameter combinations $\mathrm{C} 1$ to $\mathrm{C} 4$ and the nominal (Nom.) case, $\mathrm{K}=8.4 \times 10^{5} \mathrm{~J} / \mathrm{m}^{3}$, $M_{S}=1.1 \times 10^{6} \mathrm{~A} / \mathrm{m}$. It should be pointed out that 50 realizations have been tested for each combination and all of them resulted in successful switching. 


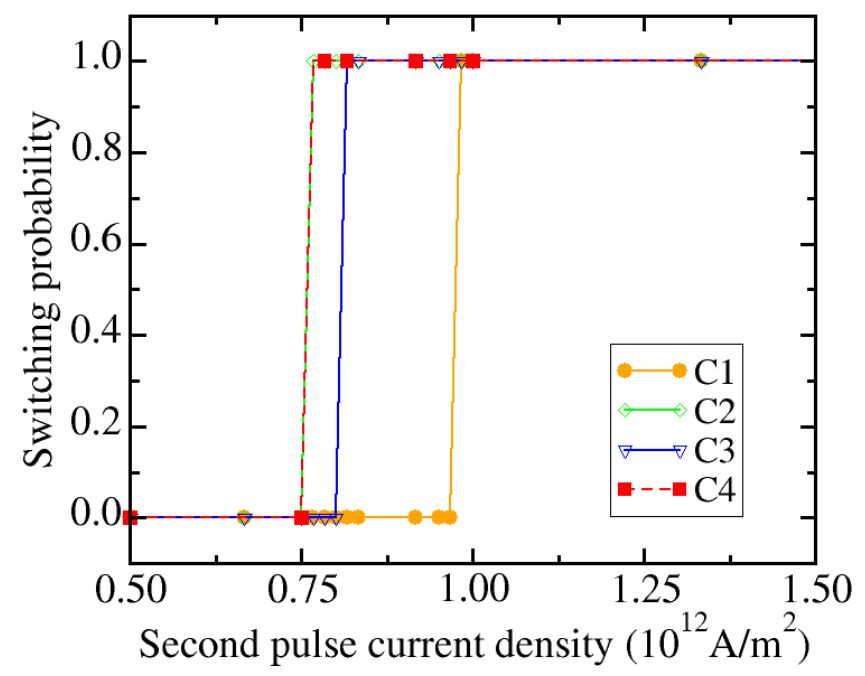

Figure 13. Switching probability as a function of the current density for the second current pulse, for four combinations of anisotropy energy and saturation magnetization. $\mathrm{C} 1: \mathrm{K}=8.8 \times 10^{5} \mathrm{~J} / \mathrm{m}^{3}$, $M_{S}=1.05 \times 10^{6} \mathrm{~A} / \mathrm{m} ; \mathrm{C} 2: K=8.8 \times 10^{5} \mathrm{~J} / \mathrm{m}^{3}, M_{S}=1.16 \times 10^{6} \mathrm{~A} / \mathrm{m} ; \mathrm{C} 3: K=8.0 \times 10^{5} \mathrm{~J} / \mathrm{m}^{3}$, $M_{S}=1.05 \times 10^{6} \mathrm{~A} / \mathrm{m} ; \mathrm{C} 4: K=8.0 \times 10^{5} \mathrm{~J} / \mathrm{m}^{3}, M_{S}=1.16 \times 10^{6} \mathrm{~A} / \mathrm{m}$. The current density of the first pulse is set to $3.0 \times 10^{12} \mathrm{~A} / \mathrm{m}^{2}$ and both pulse durations are $200 \mathrm{ps}$.

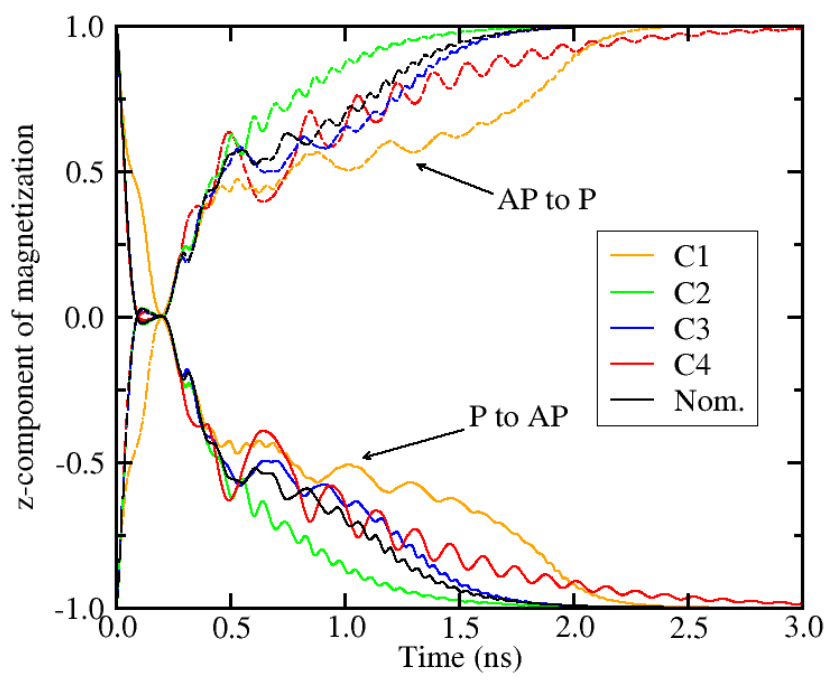

Figure 14. Average switching realizations from parallel to anti-parallel (P-AP) and anti-parallel to parallel (AP-P) for various combinations of anisotropy energy and saturation magnetization. C1: $K=8.8 \times 10^{5} \mathrm{~J} / \mathrm{m}^{3}, M_{S}=1.05 \times 10^{6} \mathrm{~A} / \mathrm{m} ; \mathrm{C} 2: K=8.8 \times 10^{5} \mathrm{~J} / \mathrm{m}^{3}, M_{S}=1.16 \times 10^{6} \mathrm{~A} / \mathrm{m} ; \mathrm{C} 3:$ $K=8.0 \times 10^{5} \mathrm{~J} / \mathrm{m}^{3}, M_{S}=1.05 \times 10^{6} \mathrm{~A} / \mathrm{m} ; \mathrm{C} 4: K=8.0 \times 10^{5} \mathrm{~J} / \mathrm{m}^{3}, M_{S}=1.16 \times 10^{6} \mathrm{~A} / \mathrm{m}$; Nom.: $K=8.4 \times 10^{5} \mathrm{~J} / \mathrm{m}^{3}, M_{S}=1.1 \times 10^{6} \mathrm{~A} / \mathrm{m}$. Each curve represents the average of 50 realizations, all of them resulting in successful switching.

\section{Conclusions}

We developed a reinforcement learning approach in combination with micromagnetic simulations to optimize the switching of a spin-orbit torque memory cell. The magnetization switching is accomplished with a two-current pulse scheme and it is shown that, for sub-nanosecond operation, the switching probability strongly depends on the parameters of the applied current pulses. We demonstrated that the reinforcement learning setup can determine optimal sequence and timing parameters for the current pulses, which results in the fastest switching of the memory cell. This optimal pulse sequence yielded a switching time as short as 146 ps, remarkably shorter in comparison to a switching time of 300 ps for the manually configured pulse sequence. Based on our results, reinforcement learning 
is a promising tool to automate and further optimize spin-orbit torque switching based on the two-pulse scheme. We analyzed the impact of material parameter variations and showed that reliable switching can be guaranteed in the presence of cell-to-cell variations, provided that the current amplitude of the pulses is adjusted.

Author Contributions: Conceptualization, R.L.d.O. and J.E.; Data curation, R.L.d.O. and J.E.; Formal analysis, R.L.d.O. and J.E.; Funding acquisition, S.S. and V.S.; Investigation, R.L.d.O. and J.E.; Methodology, R.L.d.O. and J.E.; Project administration, V.S.; Software, R.L.d.O. and J.E.; Supervision, S.S. and V.S.; Visualization, R.L.d.O. and J.E.; Writing—original draft, R.L.d.O.; Writing—review \& editing, J.E., S.F., W.G., S.S., and V.S. All authors have read and agreed to the published version of the manuscript.

Funding: This research was funded by the Christian Doppler Forschungsgesellschaft, grant number 1558669. The APC was funded by the TU Wien Library through its Open Access Funding Program.

Acknowledgments: The financial support by the Austrian Federal Ministry for Digital and Economic Affairs and the National Foundation for Research, Technology and Development is gratefully acknowledged. The authors acknowledge TU Wien Library for financial support through its Open Access Funding Program.

Conflicts of Interest: The authors declare no conflict of interest.

\section{References}

1. Jew, T. MRAM in Microcontroller and Microprocessor Product Applications. In Proceedings of the 2020 IEEE International Electron Devices Meeting (IEDM), San Francisco, CA, USA, 12-18 December 2020; pp. 11.1.1-11.1.4.

2. Han, S.; Lee, J.; Shin, H.; Lee, J.; Suh, K.; Nam, K.; Kwon, B.; Cho, M.; Lee, J.; Jeong, J.; et al. 28-nm 0.08 $\mathrm{mm}^{2}$ /Mb Embedded MRAM for Frame Buffer Memory. In Proceedings of the 2020 IEEE Electron Devices Meeting (IEDM), San Francisco, CA, USA, 12-18 December 2020; pp. 11.2.1-11.2.4.

3. Naik, V.B.; Yamane, K.; Lee, T.Y.; Kwon, J.H.; Chao, R.; Lim, J.; Chung, N.L.; Behin-Aein, B.; Hau, L.Y.; Zeng, D.; et al. JEDECQualified Highly Reliable 22nm FD-SOI Embedded MRAM For Low-Power Industrial-Grade, and Extended Performance Towards Automotive-Grade-1 Applications. In Proceedings of the 2020 IEEE Electron Devices Meeting (IEDM), San Francisco, CA, USA, 12-18 December 2020; pp. 11.3.1-11.3.4.

4. Shih, Y.C.; Lee, C.F.; Chang, Y.A.; Lee, P.H.; Lin, H.J.; Chen, Y.L.; Lo, C.-P.; Lin, K.F.; Chiang, T.W.; Lee, Y.J.; et al. A Reflow-Capable, Embedded 8Mb STT-MRAM Macro with 9ns Read Access Time in 16nm FinFet Logic CMOS Process. In Proceedings of the 2020 IEEE Electron Devices Meeting (IEDM), San Francisco, CA, USA, 12-18 December 2020; pp. 11.4.1-11.4.4.

5. Edelstein, D.; Rizzolo, M.; Sil, D.; Dutta, A.; DeBrosse, J.; Wordeman, M.; Arceo, A.; Chu, I.C.; Demarest, J.; Edwards, E.R.J.; et al. A $14 \mathrm{~nm}$ Embedded STT-MRAM CMOS Technology. In Proceedings of the 2020 IEEE Electron Devices Meeting (IEDM), San Francisco, CA, USA, 12-18 December 2020; pp. 11.5.1-11.5.4.

6. Lee, T.Y.; Yamane, K.; Otani, Y.; Zeng, D.; Kwon, J.; Lim, J.H.; Naik, V.B.; Hau, L.Y.; Chao, R.; Chung, N.L.; et al. Advanced MTJ Stack Engineering of STT-MRAM to Realize High Speed Applications. In Proceedings of the 2020 IEEE Electron Devices Meeting (IEDM), San Francisco, CA, USA, 12-18 December 2020; pp. 11.6.1-11.6.4.

7. Apalkov, D.; Dieny, B.; Slaughter, J.M. Magnetoresistive Random Access Memory. Proc. IEEE 2016, 104, 1796-1830. [CrossRef]

8. Hu, G.; Nowak, J.J; Gottwald, M.G.; Brown, S.L.; Doris, B.; D’Emic, C.P.; Hashemi, P.; Houssameddine, D.; He, Q.; Kim, D.; et al. Spin-Transfer Torque MRAM with Reliable 2 ns Writing for Last Level Cache Applications. In Proceedings of the 2019 IEEE Electron Devices Meeting (IEDM), San Francisco, CA, USA, 7-11 December 2019; pp. 2.6.1-2.6.4.

9. Alzate, J.G.; Arslan, U.; Bai, P.; Brockman, J.; Chen, Y.J.; Das, N.; Fischer, K.; Ghani, T.; Heil, P.; Hentges, P.; et al. 2 MB Array-Level Demonstration of STT-MRAM Process and Performance Towards L4 Cache Applications. In Proceedings of the 2019 IEEE Electron Devices Meeting (IEDM), San Francisco, CA, USA, 7-11 December 2019; pp. 2.4.1-2.4.4.

10. Sakhare, S.; Perumkunnil, M.; Bao, T.H.; Rao, S.; Kim, W.; Crotti, D.; Yasin, F.; Couet, S.; Swerts, J.; Kundu, S.; et al. Enablement of STT-MRAM as Last Level Cache for the High Performance Computing Domain at the 5nm Node. In Proceedings of the 2018 IEEE Electron Devices Meeting (IEDM), San Francisco, CA, USA, 1-5 December 2018; pp. 18.3.1-18.3.4.

11. Aggarwal, S.; Almasi, H.; DeHerrera, M.; Hughes, B.; Ikegawa, S.; Janesky, J.; Lee, H.K.; Lu, H.; Mancoff, B.; Nagel, K.; et al. Demonstration of a Reliable 1Gb Standalone Spin-Transfer Torque MRAM for Industrial Applications. In Proceedings of the 2019 IEEE Electron Devices Meeting (IEDM), San Francisco, CA, USA, 7-11 December 2019; pp. 2.1.1-2.1.4.

12. Sato, H.; Honjo, H.; Watanabe, T.; Niwa, M.; Koike, H.; Miura, S.; Saito, T.; Inoue, H.; Nasuno, T.; Tanigawa, T.; et al. 14ns Write Speed $128 \mathrm{Mb}$ Density Embedded STT-MRAM with Endurance $>10^{10}$ and 10yrs Retention $85^{\circ} \mathrm{C}$ Using Novel Low Damage MTJ Integration Process. In Proceedings of the 2018 IEEE Electron Devices Meeting (IEDM), San Francisco, CA, USA, 1-5 December 2018; pp. 27.2.1-27.2.4. 
13. Golonzka, O.; Alzate, J.G.; Arslan, U.; Bohr, M.; Bai, P.; Brockman, J.; Buford, B.; Connor, C.; Das, N.; Doyle, B.; et al. MRAM as Embedded Non-Volatile Memory Solution for 22FFL FinFet Technology. In Proceedings of the 2018 IEEE Electron Devices Meeting (IEDM), San Francisco, CA, USA, 1-5 December 2018; pp. 18.1.1-18.1.4.

14. Miron, I.M.; Gaudin, G.; Auffret, S.; Rodmacq, B.; Schuhl, A.; Pizzini, S.; Vogel, J.; Gambardella, P. Current-Driven Spin Torque Induced by the Rashba Effect in a Ferromagnetic Metal Layer. Nat. Mater. 2010, 9, 230-234. [CrossRef] [PubMed]

15. Honjo, H.; Nguyen, T.V.A.; Watanabe, T.; Nasuno, T.; Zhang, C.; Tanigawa, T.; Miura, S.; Inoue, H.; Niwa, M.; Yoshiduka, T.; et al. First Demonstration of Field-Free SOT-MRAM with 0.35 ns Write Speed and 70 Thermal Stability under $400{ }^{\circ} \mathrm{C}$ Thermal Tolerance by Canted SOT Structure and its Advanced Patterning/SOT Channel Technology. In Proceedings of the 2019 IEEE Electron Devices Meeting (IEDM), San Francisco, CA, USA, 7-11 December 2019; pp. 28.5.1-28.5.4.

16. Garello, K.; Yasin, F.; Hody, H.; Couet, S.; Souriau, L.; Sharifi, S.H.; Swerts, J.; Carpenter, R.; Rao, S.; Kim, W.; et al. Manufacturable $300 \mathrm{~mm}$ Platform Solution for Field-Free Switching SOT-MRAM. In Proceedings of the 2019 IEEE Symposium on VLSI Circuits, Kyoto, Japan, 9-14 June 2019; pp. T194-T195.

17. Garello, K.; Yasin, F.; Couet, S.; Souriau, L.; Swerts, J.; Rao, S.; Van Beek, S.; Kim, W.; Liu, E.; Kundu, S.; et al. SOT-MRAM 300 mm Integration for Low Power and Ultrafast Embedded Memories. In Proceedings of the 2018 IEEE Symposium on VLSI Circuits, Honolulu, HI, USA, 18-22 June 2018; pp. 81-82.

18. Fukami, S.; Anekawa, T.; Zhang, C.; Ohno, H. A Spin-Orbit Torque Switching Scheme with Collinear Magnetic Easy Axis and Current Configuration. Nat. Nanotechnol. 2016, 11, 621-626. [CrossRef] [PubMed]

19. Fukami, S.; Zhang, C.; DuttaGupta, S.; Kurenkov, A.; Ohno, H. Magnetization Switching by Spin-Orbit Torque in an Antiferromagnet-Ferromagnet Bilayer System. Nat. Mater. 2016, 15, 535-541. [CrossRef] [PubMed]

20. Oh, Y.W.; Baek, S.H.C.; Kim, Y.M.; Lee, H.Y.; Lee, K.D.; Yang, C.G.; Park, E.S.; Lee, K.S.; Kim, K.W.; Go, G.; et al. Field-Free Switching of Perpendicular Magnetization through Spin-Orbit Torque in Antiferromagnet/Ferromagnet/Oxide Structures. Nat. Nanotechnol. 2016, 11, 878-884. [CrossRef] [PubMed]

21. Wu, H.; Razavi, S.A.; Shao, Q.; Li, X.; Wong, K.L.; Liu, Y.; Yin, G.; Wang, K.L. Spin-Orbit Torque from a Ferromagnetic Metal. Phys. Rev. B 2019, 99, 184403. [CrossRef]

22. MacNeill, D.; Stiehl, G.M.; Guimaraes, M.H.D.; Buhrman, R.A.; Park, J.; Ralph, D.C. Control of Spin-Orbit Torques through Crystal Symmetry in WTe2/Ferromagnet Bilayers. Nat. Phys. 2016, 13, 300-305. [CrossRef]

23. Yu, G.; Upadhyaya, P.; Fan, Y.; Alzate, J.G.; Jiang, W.; Wong, K.L.; Takei, S.; Bender, S.A.; Chang, L.T.; Jiang, Y.; et al. Switching of Perpendicular Magnetization by Spin-Orbit Torques in the Absence of External Magnetic Fields. Nat. Nanotechnol. 2014, 9, 548-554. [CrossRef] [PubMed]

24. Sverdlov, V.; Makarov, A.; Selberherr, S. Two-Pulse Sub-ns Switching Scheme for Advanced Spin-Orbit Torque MRAM. Solid-State Electron. 2019, 155, 49-56. [CrossRef]

25. de Orio, R.L.; Ender, J.; Fiorentini, S.; Goes, W.; Selberherr, S.; Sverdlov, V. Numerical Analysis of Deterministic Switching of a Perpendicularly Magnetized Spin-Orbit Torque Memory Cell. IEEE J. Electron Devices Soc. 2021, 9, 61-67. [CrossRef]

26. Mehta, P.; Bukov, M.; Wang, C.H.; Day, A.G.; Richardson, C.; Fisher, C.K.; Schwab, D.J. A High-Bias, Low-Variance Introduction to Machine Learning for Physicists. Phys. Rep. 2019, 810, 1-124. [CrossRef] [PubMed]

27. Kovacs, A.; Fischbacher, J.; Oezelt, H.; Gusenbauer, M.; Exl, L.; Bruckner, F.; Suess, D.; Schrefl, T. Learning Magnetization Dynamics. J. Magn. Magn. Mater. 2019, 491, 165588. [CrossRef]

28. Exl, L.; Fischbacher, J.; Kovacs, A.; Oezelt, H.; Gusenbauer, M.; Yokota, K.; Shoji, T.; Hrkac, G.; Schrefl, T. Magnetic Microstructure Machine Learning Analysis. J. Phys. Mater. 2019, 2, 014001. [CrossRef]

29. Dai, M.; Hu, J.-M. Field-Free Spin-Orbit Torque Perpendicular Magnetization Switching in Ultrathin Nanostructures. NPJ Comput. Mater. 2020, 6, 78. [CrossRef]

30. Sutton, R.S.; Barto, A.G. Reinforcement Learning: An Introduction, 2nd. ed.; The MIT Press: Cambridge, MA, USA, 2018.

31. Mnih, V.; Silver, D.; Rusu, A.A.; Veness, J.; Bellemare, M.G.; Graves, A.; Riedmiller, M.; Fidjeland, A.K.; Ostrovski, G.; Petersen, S.; et al. Human-Level Control Through Deep Reinforcement Learning. Nature 2015, 518, 529-533. [CrossRef] [PubMed]

32. Silver, D.; Hubert, T.; Schrittwieser, J.; Antonoglou, I.; Lai, M.; Guez, A.; Lanctot, M.; Sifre, L.; Kumaran, D.; Graepel, T.; et al. A General Reinforcement Learning Algorithm that Masters Chess, Shogi, and Go Through Self-Play. Science 2018, 362, 1140-1144. [CrossRef] [PubMed]

33. de Orio, R.L.; Makarov, A.; Goes, W.; Ender, J.; Fiorentini, S.; Sverdlov, V. Two-Pulse Magnetic Field-Free Switching Scheme for Perpendicular SOT-MRAM with a Symmetric Square Free Layer. Phys. B Condens. Matter 2020, 578, 411743. [CrossRef]

34. Makarov, A. Modeling of Emerging Resistive Switching Based Memory Cells. Ph.D. Thesis, Institute for Microelectronics, TU Wien, Austria, 2014. Available online: https:/ /www.iue.tuwien.ac.at/phd/makarov/ (accessed on 12 March 2021).

35. Raffin, A.; Hill, A.; Ernestus, M.; Gleave, A.; Kanervisto, A.; Dormann, N. Stable Baselines 3. Available online: https://github. com/DLR-RM/stable-baselines3 (accessed on 12 March 2021). 